\title{
MACHINE LEARNING CLASSIFICATION OF COMPLEX VASCULATURE STRUCTURES FROM IN-VIVO BONE MARROW 3D DATA
}

\author{
R. A. Khorshed ${ }^{1}$ and C. Lo Celso ${ }^{1}$ \\ 1- Department of Life Sciences, Imperial College London, SW7 2AZ, UK
}

\begin{abstract}
Blood vessels inside the bone marrow (BM) play a vital role in the maintenance of hematopoietic stem cell (HSCs). Investigating the interaction of HSCs relative to vasculature has become the main headline for many recent studies. Advances in microscopy and image analysis using mouse models have allowed detection, identification and automated quantification of HSCs alongside their vascular niche. This resulted in new hypotheses concerning the activation state of HSCs adjacent to different blood vessel types (for example sinusoids vs. arterioles). Identifying the different types of BM vasculature has become critically important, however it still requires the use of complex immuno-stainings ex vivo or transgenic reporter mouse lines in vivo. To eliminate these requirements and increase the throughput of studies focusing on the HSC niche, we present a machine learning classification approach based on the Decision Tree Classifier to classify different regions of bone marrow vasculature into four distinct classes based on their discriminative features.
\end{abstract}

Index Terms - Decision Tree Classifier, classification, vasculature, bone marrow, 3D images

\section{INTRODUCTION}

Hematopoietic stem cells (HSCs) maintain the turnover of red blood cells, platelets and immune cells throughout our lifetime thanks to their ability to self-renew (maintain themselves) and differentiate. They reside in the bone marrow (BM) in a complex regulatory environment called niche, which regulates their function [1], [2]. The molecular and cellular components of the HSC niche are object of intense study as the clue to prevent and treat haematological diseases. Blood vessels are one of the currently most studied HSC niche components. Vessels have been known for decades to supply oxygen and nutrition across a living tissue; however, in recent studies BM vascular and peri-vascular cells were shown to have a direct impact on HSC maintenance and function. The majority of $\mathrm{BM}$ vasculature consists of a complex network of thin-walled, fenestrated sinusoidal capillaries (sinusoids), where HSCs proliferate, differentiate and enter circulation. Recent studies indicated that HSCs are also located close to arterioles surrounded by nestin GFP expressing cells [3], [4]. Therefore, the exact role of different vessel types in HSC function and self-renewal is far from being well understood.The visualization of the vascular niche using 3D imaging is an expanding and promising field that provides a unique perspective of HSC position in relation to vasculature [5], [6]. However, defining the variable components of the BM vascular network is a time consuming and challenging task due to the complex and interlinked structure of blood vessels and it is currently dependent on complex ex vivo immuno-stainings or, for in vivo studies, on the use of limited transgenic reporter mouse strains.

In this paper we propose a classification approach using the Decision Tree Classifier to define novel distinctive features of the variable types of blood vessels and classify them based on their morphological and topological characteristics across the BM space. A staining/reporterindependent computerized classification approach of blood vessels would considerably simplify experimental set ups, reduce the number of animals used, and allow studying further BM components alongside the vessels themselves.

\section{MATERIAL}

Wild type mice were anesthetized, their scalp removed and replaced with an imaging window [6]. To label blood vessels mice were injected i.v. with $50 \mu \mathrm{l}$ of $40 \mathrm{mg} / \mathrm{ml}$ TRITCdextran. Intravital microscopy of calvarium BM blood vessels was performed using a Zeiss LSM 780 upright confocal/two-photon combined microscope as previously described [5], [6].

In this paper we use 9 datasets, each representing a large area of calvarium BM. All datasets are encoded at 8 bits per pixel, with sizes for $X Y$ dimensions of up to 4206 by 3281 pixels, and the $Z$ dimension ranging between 43-58 slices acquired with a step size of $5 \mu \mathrm{m}$, with each slice corresponding to 1 voxel in the $Z$ dimension. Particular regions of the calvarium $\mathrm{BM}$ such as the bifurcation of central suture and coronal suture are used as reference points for alignment during the data acquisition. To train the classifier, one dataset was used to generate object samples (337 samples total for all proposed classes). The remaining 8 datasets were used for testing the classifier, together with the training dataset. 


\section{METHOD}

We use a fully automated approach for detecting and segmenting the vasculature. The LH-SEG algorithm segments regions of blood vessels using a combination of multi-resolution segmentation and mean intensity difference to neighbor thresholds as described in our previous work [7]. Once the regions of vasculature are segmented on each individual 2D slices, the $2 \mathrm{D}$ slices are linked across the $\mathrm{Z}$ dimension to form the 3D structure of vasculature, see Fig. 1. The Jaccard similarity index was used to evaluate the LHSEG segmentation of vasculature against the manual benchmarking and scored an accuracy of 0.84 which is a key point for the success of the classifier.
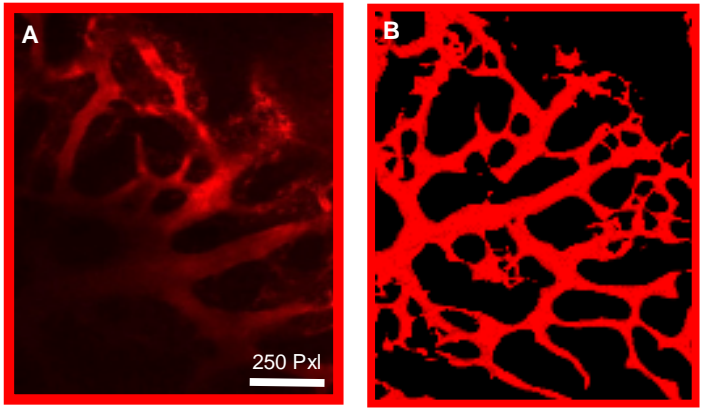

Fig.1. A) 2D raw microscopy image of BM blood vessels. B) $3 \mathrm{D}$ rendering of segmented $3 \mathrm{D}$ stack.

\subsection{Decision Tree classification}

Although the segmentation approach detects the regions of blood vessels distributed across the BM efficiently, the distinction of the multiple vasculature components is not achievable using only segmentation methods and therefore a more sophisticated method is required.

In this paper we propose the use of a supervised objectbased machine learning classification approach [8], [9]. Classification methods for image analysis are structured approaches, which build classification models from an input dataset and predict the output of an unknown dataset. We select the Decision Tree Classifier to generate the classification output for our image datasets. Decision Tree Classifier is a classification technique that can easily be applied to real life scenarios; therefore it is ideal for solving classification problems. Results can also be easily interpreted compared to other classifiers which only provide statistical comparisons. The tree starts from the top node "root", and splits into a number of nodes ("leafs") based on a number of feature vectors. Feature selection or best split is found using the criterion "purity"; if a particular feature produces a pure subset the splitting stops, otherwise the classifier iterates leaf nodes until a pure subset is found. Entropy measures the impurity of a particular subset and is defined as:

$$
E(S)=-\sum_{c \in C} p(c) \log _{2} p(c)
$$

where, $S$ is the dataset for which the entropy is calculated, $C$ the set of classes in set $S, p(c)$ the proportion of the number of elements belonging to class $C$ to the number of elements in set $S$. Pure subsets will generate a value of 0 while impure subsets will generate a value of 1
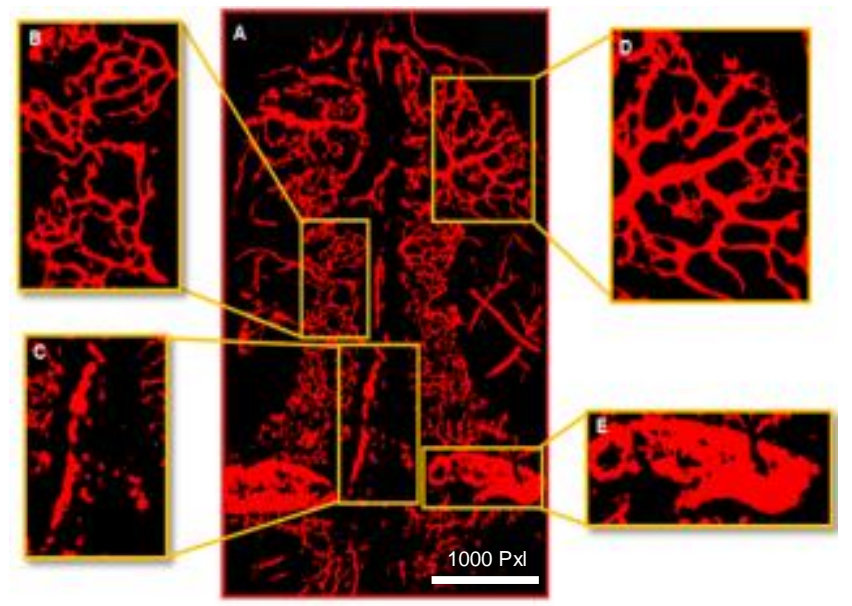

Fig.2 A) segmented and 3D rendered vasculature from a large BM area. B) vasculature area representing small sinusoids. C) vasculature area representing the central sinus. D) vasculature area representing large sinusoids. E) vasculature area representing the bifurcation of the central sinus. Since the entropy only calculates the purity of a single subset, the purity of all subsets belonging to an entire split can be computed using the information gain. The information gain calculates the average purity for all subsets resulting from a split weighted by their size as in (2);

$$
I(S, F)=\sum_{i} \frac{\left|S_{i}\right|}{|S|} \cdot E\left(S_{i}\right)
$$

where, $F$ is a feature. When a feature $F$ splits the set $S$ into subsets $S_{i}$ the average entropy is computed and the sum compared to the entropy of the original set $S$;

$$
\operatorname{Gain}(S, F)=E(S)-I(S, F)=E(S)-\sum_{i} \frac{\left|S_{i}\right|}{|S|} \cdot E\left(S_{i}\right)
$$

\subsection{Training and testing the classifier}

As blood vessels share common characteristics in terms of their complexity and interlinking, selecting effective thresholds for classifying them into different regions based on morphology is a challenging task, and difficult to solve manually as demonstrated in Fig. 2. Therefore we manually prepared a training set containing 4 classes of blood vessels objects, based on the morphological characteristics and position in relation to the BM space (Fig. 2). The first class, large sinusoids, comprises blood vessels larger in diameter and located mainly towards the periphery of the BM space. 
The second class, small sinusoids, comprises blood vessels smaller in shape, located all across the bone marrow space. The third class, central sinus, is mainly located in the middle of the bone marrow space. The fourth class, bifurcation of the central sinus, results from the central sinus branching out towards the left and right sides of the BM space. For the training task, 2D objects from blood vessels regions belonging to each class were fed to the training set. For instance, 2D objects from small interconnected sinusoids regions were selected to feed the training set for class "small sinusoids". The same was done for all remaining training classes, Fig. 3. The number of samples was optimized for each class to avoid under-fitting and over-fitting the classifier. 3-fold cross validation was used in this experiment. The size of each training set correlated with the heterogeneity of the objects within each class. Since the Decision Tree Classifier does not require a feature optimization task prior to classification, we could use a vast number of morphological and position features to train the classifier (Definiens Developer XD 64, shape and position feature list). The classifier automatically selected the discriminative features and the boundaries that defined the different classes of blood vessels objects. The Training stopped when satisfactory results were attained.

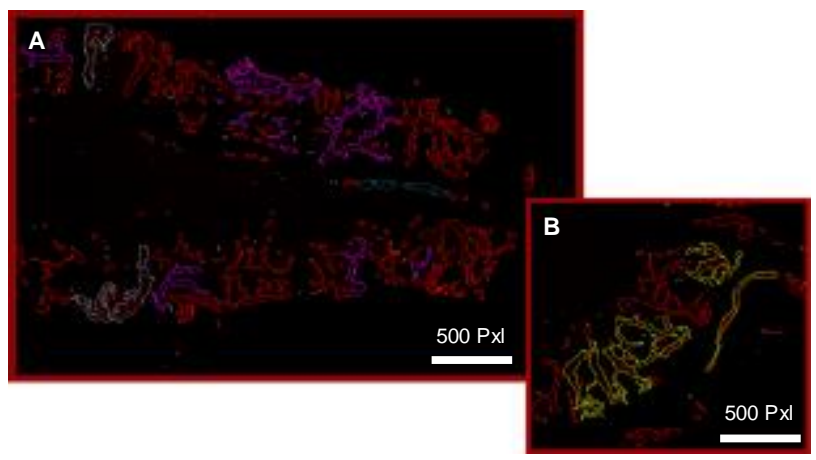

Fig.3. 2D representation of segmented blood vessels from which the training sets were generated for each class. A) Contains 3 vessel classes: vessels with magenta border represent training data for small sinusoids, blue border is for the bifurcation of the central sinus, green border is for the central sinus and red border are vessels that were left for testing. B) Vessels with yellow border represent training data for the large sinusoids. To test whether the classifier could deal with mouse-to-mouse variability, all training samples for all 4 classes were taken from one image dataset only. We then tested the classifier on all 9 remaining datasets including the dataset used for training.

\section{RESULTS}

\subsection{Classification results}

The Decision Tree Classifier selected a number of discriminative features that best classified the different types of blood vessels. Object position features; ( $\mathrm{X}$ and $\mathrm{Z}$ positions), were selected by the Decision Tree to split the root node. Further morphological features; (Width, Width/Length and Area) that described the shape of objects where then integrated into the tree to create further splits and resulted in the differentiation of classes, Fig. 4.

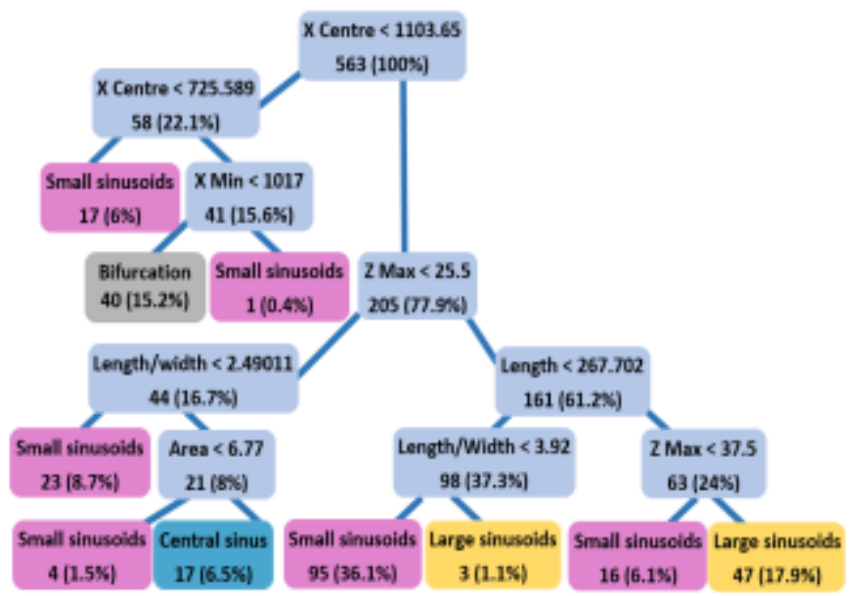

Fig.4. Results of the Decision Tree Classifier splits and feature selection after the training.

This indicates that the Decision Tree Classifier does not only classifying blood vessels components into further classes based on their feature differences, but it is also capable of selecting novel characteristics that best describe each class within the blood vessel regions, results represented in Fig.5. This is a promising result that indicates the possibility to identify different BM vessel types without the need for complex immuno-staining or transgenic reporter lines.

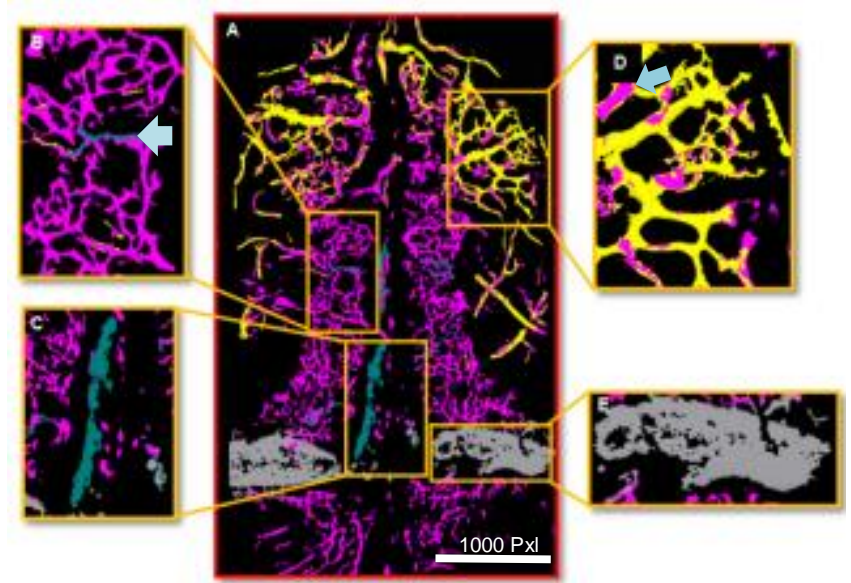

Fig.5 A) Classification of vessels from the image presented in Fig. 2 following Decision Tree Classifier analysis. B) classification results of small sinusoids (magenta). C) classification results of the central sinus (petrol blue). D) classification results of the large sinusoids (yellow). E) classification results of the bifurcation of the central sinus (grey). Arrows: examples of classification errors. 


\subsection{Classification Accuracy}

To evaluate the performance of the classification, we generate our benchmarking data by manually classifying all segmented regions of blood vessels into the four described classes (large sinusoids, small sinusoid, central sinus and the bifurcation of the central sinus). We then evaluate the results of automatically classified regions by calculating the Precision and Recall values defined in expression (4) (5) for each class.

$$
\text { Precision }=\frac{T P}{T P+F P} \quad \text { (4) } \quad \text { Recall }=\frac{T P}{T P+F N}
$$

Where $T P$ is the sum of objects volume belonging to correctly classified regions when compared to the benchmarking data, $F P$ is the sum of objects volume belonging to classified regions that did not match the benchmarking data, $F N$ is the sum of objects volume belonging to classified regions that were missed compared to the benchmarking data. The mean classification Precision and Recall for all tested datasets for each class is presented in Fig. 6. Classification results accuracy varies from one class to another depending on the complexity of the object structure. For instance, the classification of large sinusoids and bifurcation of the central sinus yields high precision and recall due to their larger size compared to objects in other classes. This ultimately results in improved distinction from other objects. In contrast, structures in the central sinus and small sinusoids classes yield slightly reduced precision and recall due to their complex, interlinked structure, which leads to misclassification of a fraction of objects.
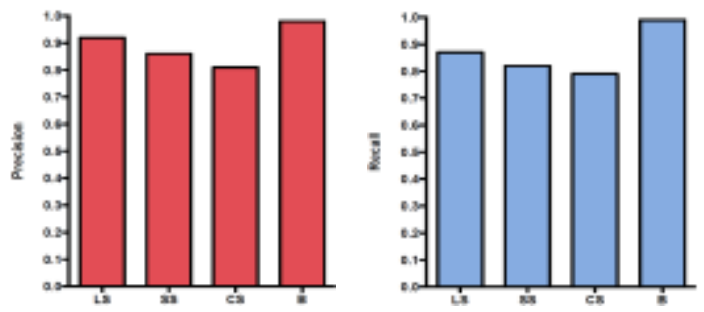

Fig.6 Precession (A) and Recall (B) results of the proposed classification approach based on all tested 9 datasets. LS: large sinusoids, SS: Small sinusoids, CS: central sinus, B: Bifurcation of the central sinus.

\section{CONCLUSION}

We propose an approach for classifying the different regions of vasculature in the bone marrow, and identify them as separate classes based on their intrinsic morphological and position features. The presented approach is based on a Decision Tree machine learning classifier, which takes into account a training set including objects from each class and a number of morphological and location features. The classifier divides all objects from all tested datasets based on these discriminative values into one of the classes (Large sinusoids, Small sinusoids, Central sinus and bifurcation of the central sinus). The classifier provides a good level of classification for all classes and is more time efficient than manual classification. More complex objects such as small sinusoids and central sinus yield slightly higher error rate, therefore further training cycles from multiple datasets might be needed to account for the complexity and variability of such objects. Further development could include classifying the larger sinusoids into additional two classes, as at present they may be a mixture of sinusoids and arterioles. Further classifiers could also be investigated to evaluate whether the performance of this approach could be further improved. Finally, identifying the different classes of vasculature based on their intrinsic characteristics eliminates the need for complex ex vivo and in vivo staining. This increases the throughput of intravital imaging and our understanding of the complexity of the HSC niche.

\section{REFERENCES}

[1] C. Lo Celso and D. T. Scadden, "The haematopoietic stem cell niche at a glance," J Cell Sci, vol. 124, pp. 3529-35, Nov 12011.

[2] S. J. Morrison and D. T. Scadden, "The bone marrow niche for haematopoietic stem cells," Nature, vol. 505, pp. 327-34, Jan 16 2014.

[3] A. Mendelson and P. S. Frenette, "Hematopoietic stem cell niche maintenance during homeostasis and regeneration," Nat Med, vol. 20, pp. 833-846, 08//print 2014.

[4] Daniel J. Nolan, M. Ginsberg, E. Israely, B. Palikuqi, M. G. Poulos, D. James, et al., "Molecular Signatures of Tissue-Specific Microvascular Endothelial Cell Heterogeneity in Organ Maintenance and Regeneration," Developmental Cell, vol. 26, pp. 204-219, 7/29/ 2013.

[5] C. Lo Celso, C. P. Lin, and D. T. Scadden, "In vivo imaging of transplanted hematopoietic stem and progenitor cells in mouse calvarium bone marrow," Nat Protoc, vol. 6, pp. 1-14, Jan 2011.

[6] N. M. Rashidi, M. K. Scott, N. Scherf, A. Krinner, J. S. Kalchschmidt, K. Gounaris, et al., "In vivo time-lapse imaging of mouse bone marrow reveals differential niche engagement by quiescent and naturally activated hematopoietic stem cells," Blood, May 212014.

[7] R. A. Khorshed, E. D. Hawkins, D. Duarte, M. K. Scott, O. A. Akinduro, N. M. Rashidi, et al., "Automated Identification and Localization of Hematopoietic Stem Cells in 3D Intravital Microscopy Data," Stem Cell Reports, vol. 5, pp. 139-53, Jul 14 2015.

[8] C. Agarwal and A. Sharma, "Image understanding using decision tree based machine learning," in International Conference on Information Technology and Multimedia, pp. 1-8., pp. 1-8, 2011.

[9] O. Aydemir and T. Kayikcioglu, "Decision tree structure based classification of EEG signals recorded during two dimensional cursor movement imagery," J Neurosci Methods, vol. 229, pp. 6875, May 302014. 